\title{
NUMERICAL SIMULATION OF HEAT EXCHANGE IN DISCRETE-ROUGH CHANNELS AT FLOW SUPERIMPOSED OSCILLATIONS
}

\author{
Egils Dzelzitis, Natalia Sidenko \\ Riga Technical University, Latvia \\ egils.dzelzitis@rtu.lv,nsidenko@inbox.lv
}

\begin{abstract}
At present great attention is given to a problem of research of hydrodynamics and heat exchange in pulsating flows. Experimental studies for the flow in smooth channels show that pulsation of fluid flow significantly affects heat transferring and can be accompanied by both reduction and increasing in the intensity of heat exchange. However, at the present day, there is little reliable information about the effect of a pulsating flow on heat transferring in channels with discrete roughness. Thus, detection of regularities in heat exchanging processes of such flows in discretely rough channels is an actual problem for today. In this paper the results of numerical simulation of heat exchange in smooth and discrete-rough channels with a pulsating motion of fluid in them are presented. It is revealed that in the pulsating flow the instantaneous values of the heat exchange coefficient change with the change in the instantaneous values of the Reynolds numbers. Three zones of influence of the pulsing flow on the heat exchange in the channels are established. In the first area, with the Strouhal numbers $0<S h<2$, increasing in the average value of heat exchange in $10-15 \%$ is observed. In the second area $2<S h<4.3$, its decreasing in 10-15\%. In the third area, $4.3<S h<6$, the average value of the heat exchanging value becomes almost the same as for the case of a stationary flow.
\end{abstract}

Keywords: numerical simulation, pulsating flow, Navier-Stokes formula, heat exchange, discrete roughness.

\section{Introduction}

At present there is increased interest to study the heat exchange and flow patterns in channels in the presence of hemispheric recesses (concavities). This is explained by the fact that the concavities have shown themselves to be an effective intensifier of the heat exchange during their flow around [1].

Studies of the flow in channels with intensifiers in the form of concavities were considered in the researches [2-4], where it was experimentally established that heat exchange surfaces with spherical concavities significantly (1.5-4.5 times) increase heat exchange with a moderate increase of hydraulic resistance.

Experimental studies of unsteady flows in the same way showed that the flow pulsations can significantly affect hydrodynamics and heat transfer. Such non-stationary processes can be accompanied by both an increase and a decrease of the intensity of heat exchange [1].

The available research results of turbulent pulsating flows mainly relate to the kinematic structure of the flow and heat exchange in smooth channels [1;5]. The increase in the heat exchange in smooth channels is observed at high amplitude of flow rate pulsations exceeding the average velocity.

The perspective of intensification of heat transfer due to the flow pulsations was reviewed in [78]. They studied the hydrodynamic thermal processes in separation of the pulsating flow in the flow around of the single obstacles on the wall. In the studies was found the effect of a sharp reduction in the length of the tear-off area after a single obstacle compared with the stationary mode. It is shown that the average heat exchange coefficient can increase up to $60 \%$. In this case, periodic pulsations do not cause a noticeable increasing of the hydraulic resistance of the discretely rough channels.

Earlier, the authors of the research [9] developed a basic technique for computer simulation and numerical calculation of hydraulic and thermodynamic parameters in a channel with discrete roughness in the form of hemispherical concavities. The stationary motion of a fluid in smooth and discretely rough channels was considered. The developed technique allowed to obtain results that satisfactorily coincide with the well-known theoretical calculations, thereby confirming its adequacy. Comparison of the results of theoretical solutions and numerical calculations for a smooth channel showed a sufficient approval that does not exceed the allowable range of 5-10 \%.

On the basis of the developed method [9], in this research by numerical simulation methods an attempt was made to determine the peculiarities of the influence of periodic pulsations of the flow velocity on heat exchange in a channel with discrete roughness in the shape of hemispherical concavities. 


\section{Materials and methods}

Computer simulation was carried out using the selected CAD/CFD complex of SolidWorks/Flow Simulation software. In SolidWorks CAD software the calculated three-dimensional models of smooth and discrete-rough channels of circular cross section were built.

In both cases the diameters of inlet and outlet and the length of the channel are the same. The internal diameter of the channel is $D=0.018 \mathrm{~m}$, the wall thickness is $\delta=0.002 \mathrm{~m}$. The channel length was chosen with a relatively large $L=1.6 \mathrm{~m}$ in order to exclude the influence of the initial segment on the heat exchange. As a discrete rough surface in the channel the hemispherical depressions (concavities) with a sharp entrance and exit edges located in the corridor order were selected. The relative pitch $S$ between the axes of the concavities is $S / h=10$ (the h-radius of the concavity), the concavities setting angle is $\varphi=120^{\circ}$. The walls of the channels were modeled as aluminum and heated to a temperature of $t_{\text {wall }}=105^{\circ} \mathrm{C}$. To the inlet to the channel a working fluid water was supplied with a temperature $t_{\text {inlet }}=20^{\circ} \mathrm{C}$.

In the research the model of periodically pulsating internal fluid flow with velocity pulses of sinusoidal type (1) is used (Fig.1):

$$
V(t)=0.4(1+A \sin (2 \cdot \pi \cdot f \cdot t)),
$$

where $t$-time, s;

$V$ - flow velocity, $\mathrm{m} \cdot \mathrm{s}^{-1}$

$A$ - amplitude of the pulsations $\mathrm{A}=1$;

$f$ - frequency, $\mathrm{Hz}$.

The time-averaged flow rate for all cases is the same and equal to $V_{0}=0.4 \mathrm{~m} \cdot \mathrm{s}^{-1}$.

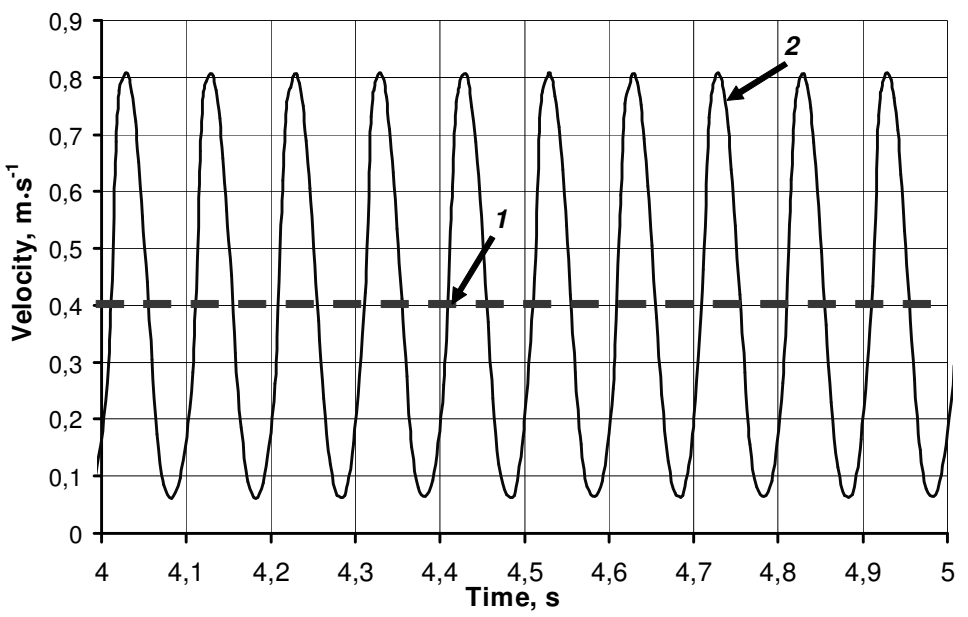

Fig. 1 . Flow velocity profile: 1 - average velocity; 2 - determined by the expression velocity (1)

For this velocity the average Reynolds number is:

$$
R e_{0}=\frac{V_{0} \cdot D}{v},
$$

where $v$ - kinematic coefficient of viscosity $v=1.006 \cdot 10^{-6}, \mathrm{~m}^{2} \cdot \mathrm{s}^{-1}$ of the water at $t_{\text {inlet }}=20^{\circ} \mathrm{C}$; $R e_{0}-$ average Reynolds number, $\operatorname{Re}_{0}=7157$.

With a change in the average Reynolds number the average velocities changed accordingly. The instantaneous values of the Reynolds numbers depend on time and for the case (1) are described by the formula:

$$
\operatorname{Re}_{t}=(1+A \sin (2 \cdot \pi \cdot f \cdot t)) \cdot \mathrm{Re}_{0},
$$

where $R e_{t}-$ Reynolds number from the time.

In the calculations the range of the flow pulsating frequency $0 \leq f<130 \mathrm{~Hz}$ was investigated. 
The non-stationary Navier-Stokes equation, the energy equation (the first law of thermodynamics) and the equation of state $[6 ; 10]$ are used for mathematical modeling of the motion of the medium and heat exchange. For turbulent flows the initial equations are averaged by the Reynolds method and additional stresses due to turbulent parameter pulsations are taken into account [6;10]. The obtained unclosed system of equations is closed with the help of additional equations for the kinetic energy of turbulence $\mathrm{k}$ and dissipation of the turbulence energy $\varepsilon$ in accordance with the known $\mathrm{k}-\varepsilon$ turbulence model [10]. The system of equations of conservation of momentum (4), mass (5) and energy (6), describing turbulent, laminar and transient flows of compressible fluid with heat exchange can be represented as:

$$
\begin{gathered}
\frac{\partial \rho u_{i}}{\partial t}+\frac{\partial}{\partial x_{j}}\left(\rho u_{i} u_{j}-\tau_{i j}\right)+\frac{\partial P}{\partial x_{i}}=F_{i} \\
\frac{\partial \rho}{\partial t}+\frac{\partial}{\partial x_{j}}\left(\rho u_{j}\right)=0 \\
\frac{\partial(\rho E)}{\partial t}+\frac{\partial}{\partial x_{i}}\left((\rho E+P) u_{i}+q_{i}-\tau_{i j} u_{j}\right)=F_{i} u_{i}+Q_{H}
\end{gathered}
$$

Two additional transport equations are used to describe the turbulent kinetic energy $k$ and dissipation $\varepsilon$ :

$$
\begin{gathered}
\frac{\partial \rho k}{\partial t}+\frac{\partial}{\partial x_{i}}\left(\rho u_{i} k\right)=\frac{\partial}{\partial x_{i}}\left(\left(\mu_{l}+\frac{\mu_{t}}{\sigma_{k}}\right) \frac{\partial k}{\partial x_{i}}\right)+S_{k} \\
\frac{\partial \rho \varepsilon}{\partial t}+\frac{\partial}{\partial x_{i}}\left(\rho u_{i} \varepsilon\right)=\frac{\partial}{\partial x_{i}}\left(\left(\mu_{l}+\frac{\mu_{t}}{\sigma_{\varepsilon}}\right) \frac{\partial \varepsilon}{\partial x_{i}}\right)+S_{\varepsilon}
\end{gathered}
$$

where the source terms $\mathrm{S}_{k}(9)$ and $\mathrm{S} \varepsilon(10)$ are defined as:

$$
\begin{gathered}
S_{k}=\tau^{R}{ }_{i j} \frac{\partial u_{i}}{\partial x_{j}}-\rho \varepsilon+\mu_{t} P_{B} \\
S_{\varepsilon}=C_{\varepsilon 1} \frac{\varepsilon}{k}\left(f_{1} \tau^{R}{ }_{i j} \frac{\partial u_{i}}{\partial x_{j}}+\mu_{t} C_{B} P_{B}\right)-C_{\varepsilon 2} f_{2} \frac{\rho \varepsilon^{2}}{k}
\end{gathered}
$$

Following Boussinesq assumption, the Reynolds-stress tensor (11) has the following form:

$$
\tau_{i j}^{R}=\mu_{t}\left(\frac{\partial u_{i}}{\partial x_{i}}+\frac{\partial u_{j}}{\partial x_{i}}-\frac{2}{3} \frac{\partial u_{l}}{\partial x_{l}} \delta_{i j}\right)-\frac{2}{3} \rho k \delta_{i j} ;
$$

The diffusive heat flux (12) is defined as:

$$
q_{i}=-\left(\frac{\mu_{l}}{\operatorname{Pr}}+\frac{\mu_{t}}{\sigma_{c}}\right) C_{P} \frac{\partial T}{\partial x_{i}} ;
$$

Upon analyzing the conjugate heat exchange between the flow and the solid, the heat transfer in the solid is simulated by the known heat transfer equation [10]:

$$
\frac{\partial \rho e}{\partial t}=\frac{\partial}{\partial x_{i}}\left[\lambda_{s} \frac{\partial T}{\partial x_{i}}\right]+Q_{H},
$$

where $u_{i j}$ - fluid velocity is a function of four independent variables $x, y, z, t$;

$P$ - fluid pressure is a function of four independent variables $x, y, z, t$;

$\rho$ - fluid density; 
$F_{i}$ - total force acting on the mass unit;

$E$ - total energy of fluid mass unit;

$Q_{H}$ - heat source per volume unit;

$\tau_{i j}$ - viscous shear stress tensor;

$i=x, y, z ; j=x, y, z-$ summing is made by subscripts;

$\mu_{l}, \mu_{t}-$ dynamic viscosity coefficient, turbulent viscosity coefficient;

$\sigma_{k}, \sigma_{\varepsilon}-$ empirical constants;

$\tau_{i j}^{R}$ - stress tensor in Reynolds model;

$g_{i}$ - components of gravitational acceleration in direction $x_{i}$;

$\mathrm{Pr}$ - Prandtl number;

$C_{P}$ - specific thermal capacity at constant pressure;

$\delta_{i j}$ - Kronecker symbol;

$e$ - specific heat;

$T$ - temperature;

$\lambda_{s}-$ solid thermal conductivity.

The above equations (4)-(13) are highly general. Here_inafter, the authors concretize the constant values as well as the dependent and independent variables to solve the particular problems.

Equations (4)-(13) are solved numerically using CFD software Flow Simulation [10]. Initial and boundary conditions for specific tasks are set in Flow Simulation software in the volume and on the corresponding surfaces of the three-dimensional design model created in CAD (computer-aided design) SolidWorks software.

For numerical solving the system of equations (4)-(13), the Flow Simulation software uses the finite volume method with an adaptive rectangular grid. In the process of calculation the initial grid of finite volumes in the computational area is crushed automatically or according to the given rule in areas of assumed large gradients of each of the dependent variables or in areas of significant change in the curvature of the solid surfaces. All basic variables are referred to mass centers of control volumes. These cell-centered values are used for approximations. The integral conservation laws may be represented in the form of the cell volume and surface integral equation (14)-(15) [10]:

$$
\frac{\partial}{\partial t} \int U d v+\oint F d s=\int Q d v
$$

are replaced by the discrete form:

$$
\frac{\partial}{\partial t}(U \cdot V)+\sum_{c e l l} F \cdot S=Q \cdot V
$$

where $F$-fluxes;

$U$ - vector of physical parameters;

$V$ - cell volume;

$S$ - cell surface area;

$Q$ - mass forces.

For reasonable accuracy of the results of the solution in this work the order of $1.000 .000-$ 1.200.000 liquid and solid elements was required.

Note that in accordance with the calculation methodology any stationary problem is initially solved as non-stationary. The solution is considered to be found after its settings of time.

The results of numerical calculations summarizing a series of computer experiments were processed in the dimensionless criteria of Reynolds Re (2), Strouhal Sh (16), and Nusselt Nu (17) [8]:

$$
S h=\frac{f \cdot D}{V_{0}},
$$

where $S h-$ dimensionless frequency of flow pulsations. 


$$
N u=\frac{\alpha \cdot D}{\lambda},
$$

where $\alpha$-heat exchange coefficient, $\mathrm{W} \cdot\left(\mathrm{m}^{2} \cdot \mathrm{K}\right)^{-1}$;

$\lambda$ - heat conduction coefficient of the liquid, $\mathrm{W} \cdot(\mathrm{m} \cdot \mathrm{K})^{-1}$.

\section{Results and discussion}

The results showed that as opposed to the stationary case with a unique dependence $N u=\varphi\left(\operatorname{Re}_{0}\right)$ in a pulsating flow the instantaneous values of the coefficient $N u(t)=\varphi\left(R e_{t}\right)$ changed with changing the instantaneous values of the Reynolds numbers $R e_{t}$ (the $\mathrm{Re}_{\mathrm{t}}$ value is determined by formula (2)). The corresponding figures for one period of oscillation of a sinusoidal pulse (1) are shown in Fig. 2, for smooth -1 and discretely rough -2 channels with frequency of pulsations of the flow $f=20 \mathrm{~Hz}$. It can be seen that each value of $\mathrm{Re}_{\mathrm{t}}$ corresponds to its own value $\mathrm{Nu}$ and is limited to its minimal $N u_{\min (1,2)}$ and maximal $N u_{\max (1,2)}$ values. The average value $N u_{\mathrm{ever}(1,2)}$ matches to half the length of the curve. This effect is due to the periodic change in the Reynolds number and the inertia of the processes during unsteady fluid flow in the channel.

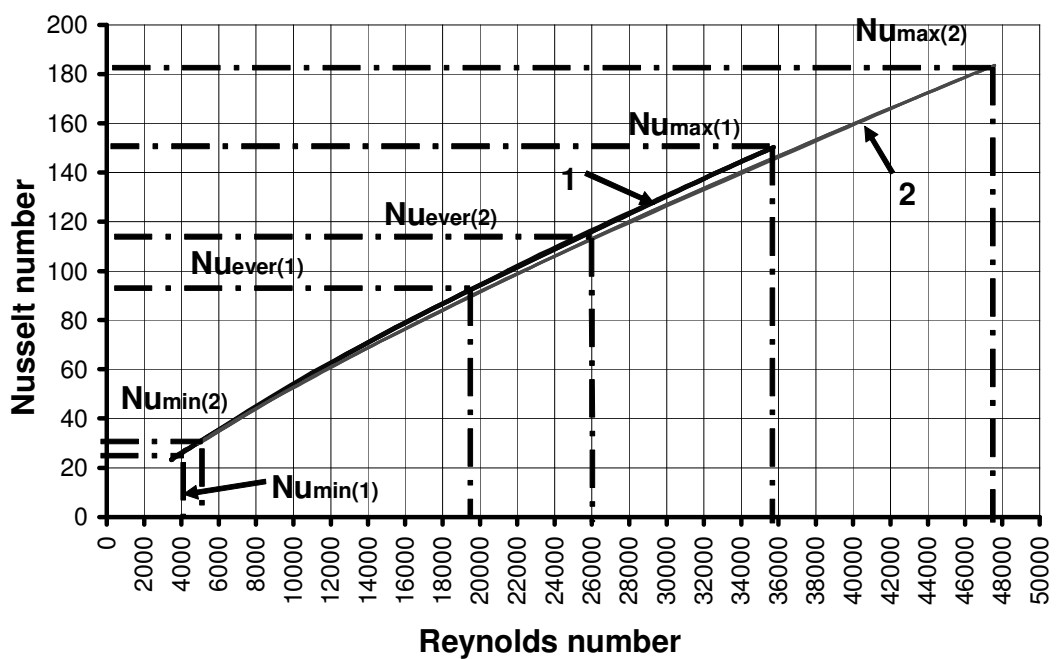

Fig. 2. Dependence of $N u(t)=\varphi\left(\operatorname{Re}_{t}\right)$ for one period of oscillation of sinusoidal pulse with $f=20 \mathrm{~Hz}: 1$ - smooth channel; 2 - discretely rough channel

The appearance of additional detachable vortices in the channel concavities in the pulsating flow causes the formation of local extremes, both on the $V(t)$ curves and on the curves $N u(t)$ (Fig. 3). As a result the $N u(t)$ curves acquire a rather complex configuration indirectly reflecting the effect of flow pulsations on heat transfer in the channel with discrete roughness. Fig. 3 shows the dependency diagram of the $V(t)$ and $N u(t) \cdot 10^{-1}$ for the frequency of the pulsations of the flow a $-f=5 \mathrm{HZ}, \mathrm{b}-$ $f=60 \mathrm{~Hz}$ in a discretely rough channel.

The fixed temperature of the channel's wall and the velocity of the incident flow lead to the change in the kinematic coefficient of viscosity of the fluid in the boundary layer, which causes a corresponding change in the Reynolds number and the frequency of vortex separation in the concavities. Thus, in the case of a channel with a discrete roughness a change in the frequency of separation and the intensity of tearing vortices in a pulsating flow as well as a predetermined temperature of the heated wall can generally affect the shape of the separation areas and the intensity of heat exchange between the channel wall and the fluid flow.

For the dependence $N u=\varphi(S h)$ we can conditionally distinguish three characteristic areas (Fig.4). In the first area, $0<S h<2$, for both channel types - smooth (index-1) and discretely rough (index -2), the frequency of the flow velocity pulsations leads to increasing in the average values of the Nusselt numbers $N u_{\text {ever(1,2) }}$ with respect to $\mathrm{Nu}_{0(1,2)}$ for the stationary mode of the fluid flow. At the same time, the minimal values of $N u_{\min (1)}$ for a smooth channel are 30-40\% lower than those of $N u_{\min (2)}$ for discretely rough and the maximal values of $N u_{\max (1)+}$, below $N u_{\max (2)}$ by approximately $20 \%$. 
a)

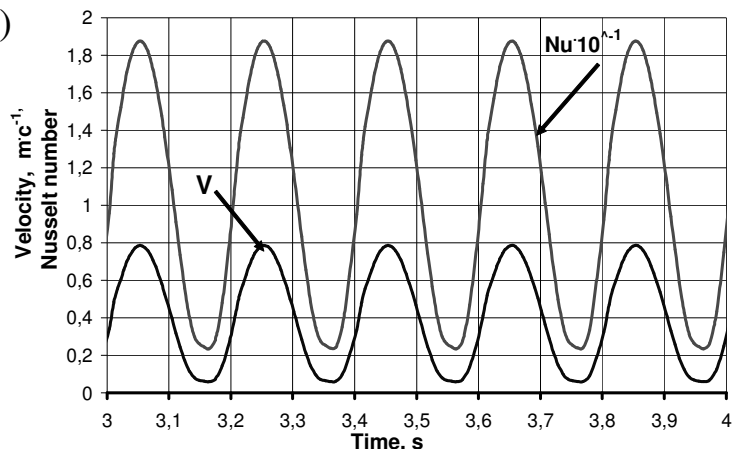

b)

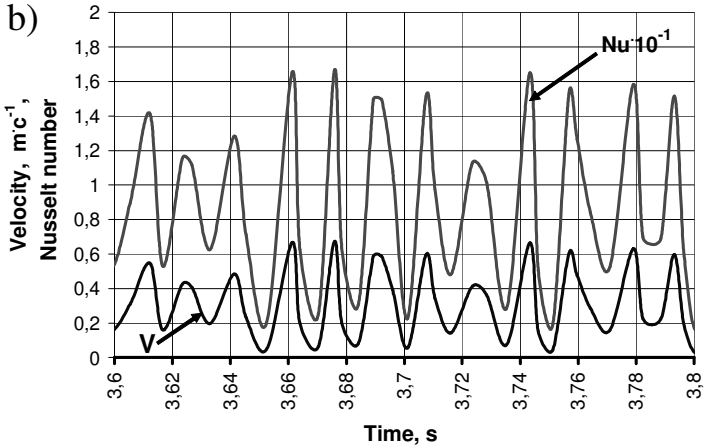

Fig. 3. Dependences of $\mathrm{V}(\mathrm{t})$ and $\mathrm{Nu}(\mathrm{t}) \cdot 10^{-1}$ for frequency of pulsations of flow in discretely rough channel: $\mathrm{a}-\mathrm{f}=5 \mathrm{HZ} ; \mathrm{b}-\mathrm{f}=60 \mathrm{HZ}$

In the second area $2<S h<4.3$ in both cases the average values of the numbers $N u_{\text {ever(1,2) }}$ become lower with respect to $N u_{0(1,2)}$ for the steady-state mode of fluid flow. And the minimal and maximal remain almost unchanged.

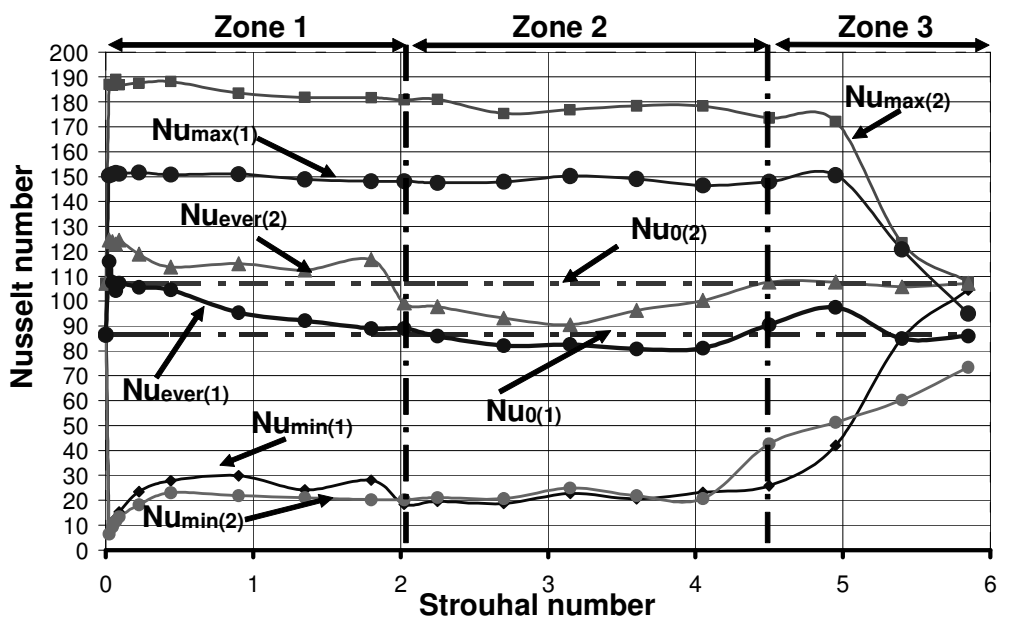

Fig. 4. Dependence of Nusselt number on dimensionless frequency of flow pulsations $N u=\varphi(S h)$
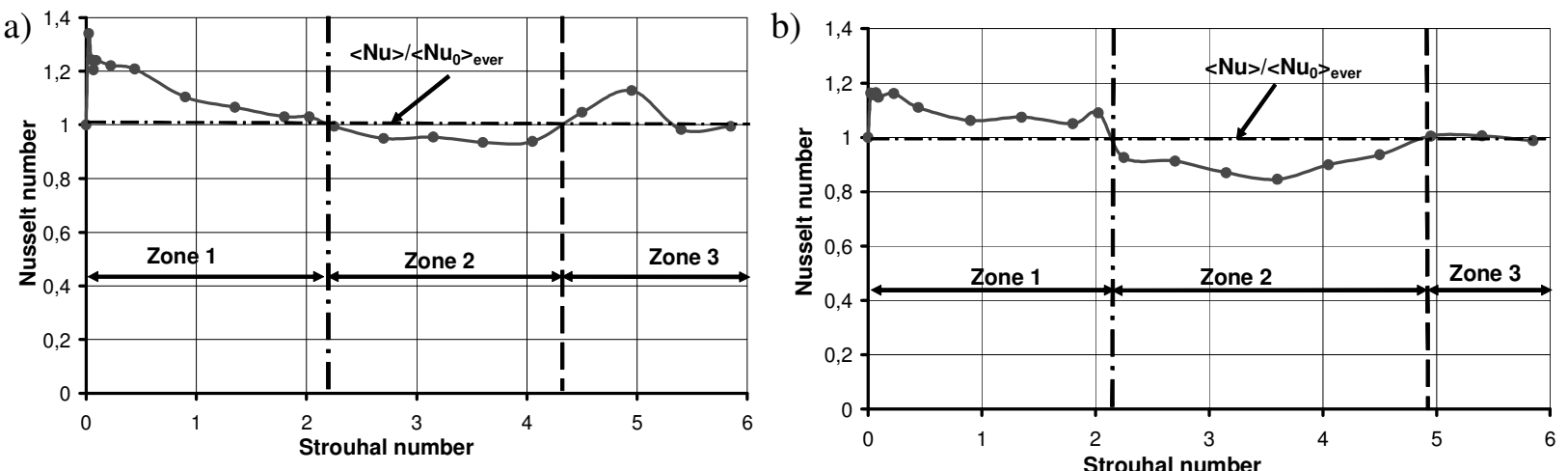

Fig. 5. Dependence of average values of Nusselt numbers on dimensionless frequency of flow pulsations $\left\langle N u>\mid<N u_{0}\right\rangle=\varphi(S h), a-$ smooth channel; $b-$ discretely rough channel

Figure 5 shows the dependence of the everage values of the Nusselt numbers $N u_{\text {ever }(1,2)}$ with respect to $N u_{0(1,2)}$ for the steady-state regime of fluid flow $\left\langle N u>\mid<N u_{0}\right\rangle=\varphi(S h)$. a - for a smooth channel $\left\langle N u_{\text {ever (1) }}\right\rangle /\left\langle N u_{0(1)}\right\rangle=\varphi(S h), \mathrm{b}-$ for a discretely rough channel $\left\langle N u_{\text {ever }(2)}\right\rangle /\left\langle N u_{0(2)}\right\rangle=\varphi(S h)$. In Fig. 5 it is vividly shown that, when reaching the dimensionless Strouhal number $S h \approx 2$ (area 2), the heat exchane in both cases worsens. And in the third area, with $4.3<S h<6$. the average value of 
$N u_{\text {ever }(1,2)}$ with respect to $N u_{0(1,2)}$ stabilizes and the value of $N u$ remains almost unchanged and the flow pulsations have virtually no effect on heat exchange.

\section{Conclusions}

1. It is shown that periodic pulsations of the flow rate of fluid in smooth and discretely rough channels have a significant effect on the heat exchange coefficient $N u$.

2. It was revealed that, in contrast to the stationary case, in a pulsating flow the instantaneous values of the coefficient $\mathrm{Nu}$ change with changing the instantaneous values of the Reynolds numbers $\mathrm{Re}_{\mathrm{t}}$.

3. The presence of three characteristic areas of the dependence of the Nusselt number $\mathrm{Nu}$ on the dimensionless Strouhal number $S h N u=\varphi(S h)$ is established. In the first area $(0<S h<2)$ the pulsations of the internal fluid flow in the channels improve the intensity of the heat exchange by about $10-15 \%$.

4. In the second area $(2<S h<4.3)$ the average values of the Nusselt number for a pulsating flow compared to the stationary one for a smooth channel decrease by $\approx 10 \%$, and for a discretely rough channel by $10-15 \%$.

5. In the third area $(4.3<S h<6)$ flow pulsations have almost no effect on heat exchange and the average value of the Nusselt number becomes approximately the same as for the stationary mode.

\section{Acknowledgements}

This work has been supported by the European Regional Development Fund within the Activity 1.1.1.2 "Post-doctoral Research Aid" of the Specific Aid Objective 1.1.1 "To increase the research and innovative capacity of scientific institutions of Latvia and the ability to attract external financing, investing in human resources and infrastructure" of the Operational Programme "Growth and Employment" (No Nr.1.1.1.2./VIAA/1/16/093).

\section{References}

[1] Galicejskij B.M. Ryzhov YU.A., Yakush E.V. Thermal and hydrodynamic processes in oscillating flows. Int:Mechanical Engineering, 1977. 256 p.

[2] Mahmood G. I., Hill M. L., Nelson D. L., Ligrani P. M., Moon H.-K., Glezer B. Local heat transfer and flow structure on and above a dimpled surface in a channel. Transactions of the ASME. Journal of Turbomachinery, 2001,vol. 123, iss. 1, pp. 115 - 123. DOI: 10.1115/1.1333694

[3] Kiselev N.A., Burtsev S.A., Strongin M.M. A procedure for determining the heat transfer coefficients of surfaces with regular relief. Measurement Techniques, 2015, vol. 58, no. 9, pp. 1016-1022. DOI: 10.1007/s11018-015-0835-7

[4] Leontiev A.I., Dilevskaya E.V., Vinogradov Yu.A., Yermolaev I.K., Strongin M.M., Bednov S.M., Golikov A.N. Effect of vortex flows at surface with hollow-type relief on heat transfer coefficients and equilibrium temperature in supersonic flow. Experimental Thermal and Fluid Science, 2002, vol. 26, iss. 5, pp. 487-497. DOI: 10.1016/S0894-1777(02)00157-7.

[5] Mizushina T., Maruyama T., Hirasava H. Strukture of the turbulence in pulsating pipe flows. Eng. Japan -1975-Vol.8, Nr.3, pp. 210-216.

[6] Chang P. Separation of Flow //New York, Pergamon Press, 1971.

[7] Saric S., Jakirlic S., Tropea C. A Periodically Perturbed Backward-Facing Step Flow by Means of LES, DES and T-RANS: An Example of Flow Separation Control // Journal of Fluids Engineering. Sept. 2005. Vol.127. pp.879-887.

[8] Mikheev N.I. Heat exchange at the separation of a pulsating flow / N.I.Miheev, I.A. Davletshin, V.M. Molochnikov // Thermal processes in engineering. 2009. F.1. №8. p.314-317.

[9] Sidenko N., Dzelzitis E. The method of numerical modeling of hydrodynamics and heat exchange in a channel with discrete roughness. International Scientific Journal „Mathematical Modeling” 2018, pp. 21-25.

[10] Alyamovsky A.A. and others. SolidWorks 2007/2008. Computer modeling in engineering practice. // BHV- Petersburg, Saint - Petersburg, 2008. 